further enhanced, but finite, conserving susceptibility at $T=0$.

Without doing the calculation, all we may do is speculate what the results will be. However, our experience with the zero-width calculation indicates that the computational problem involved is stupendous. Some approximation, such as a Lorentzian shape to the low-frequency modes, ${ }^{4,5}$ will be es- sential before such a calculation can be contemplated.

\section{ACKNOWLEDGMENTS}

We would like to thank Professor M. J. Levine, Dr. T. V. Ramakrishnan, and Professor H. Suhl for many helpful conversations during the course of this research.
${ }^{1}$ H. Suhl, Phys. Rev. Letters 19, 442 (1967); 19, 735

(E) (1967).

${ }^{2}$ P. W. Anderson, Phys. Rev. 124, 41 (1961).

${ }^{3}$ P. A. Wolff, Phys. Rev. 124, 1030 (1961).

${ }^{4}$ M. J. Levine and H. Suhl, Phys. Rev. 171, 567

(1968); M. J. Levine, T. V. Ramakrishnan, and R。A. Weiner, Phys. Rev. Letters 20, 1370 (1968).

${ }^{5}$ D. R. Hamann, Phys. Rev. 186, 549 (1969).

${ }^{6}$ We shall use the notation of the Anderson model in this paper; the results obtained are identical with those based on the Wolff model.

${ }^{7}$ M. T. Beal-Monod and D. L. Mills, Phys . Rev. Letters 24,225 (1970)。

${ }^{8}$ A. A. Abrikosov, Physics 2,5 (1965); B. Roulet, J. Gavoret, and P. Nozières, Phys. Rev, 178, 1072 (1969).

${ }^{9}$ R. A. Weiner, Phys. Rev. Letters 24, 1071 (1970).

${ }^{10} \mathrm{G}$. Baym and L. P. Kadanoff, Phys. Rev. 124, 287 (1961).

${ }^{11}$ The parquet diagrams are only a formal method of generating all diagrams for the vertex function, since the totally irreducible vertex $I$ must still be obtained by a perturbative expansion.

${ }^{12}$ A. A. Abrikosov, L. P. Gor'kov, and I. -E. Dzyaloshinski, in Methods of Quantum Field Theory in Statistical Physics, edited by R. Silverman (PrenticeHall, Englewood Cliffs, N. J., 1963).

${ }^{13}$ The Bethe-Salpeter equation for $\gamma^{e e}$ does not generate a narrow low-frequency mode.

${ }^{14}$ The solution to the Suhl vertex equation is roughly proportional to $\delta_{\omega, 0}$ at high temperatures (see M. J. Levine and H. Suhl, Ref. 4).

${ }^{15}$ This shifts the effective location of the $d$-state resonance to $\epsilon=0$ (see Ref. 5).

${ }^{16} \mathrm{~A}$ similar calculation of the susceptibility in a localmoment model has been reported by J. A. Appelbaum and D. R. Penn, Phys. Rev. B $\underline{3}, 942$ (1971).

${ }^{17} \mathrm{~J}$. R. Schrieffer and P. A. Wolff, Phys. Rev. 149, 491 (1966).

\title{
Renormalization Group and Critical Phenomena. I. Renormalization Group and the Kadanoff Scaling Picture*
}

\author{
Kenneth G. Wilson \\ Laboratory of Nuclear Studies, Cornell University, Ithaca, New York 14850 \\ (Received 2 June 1971)
}

\begin{abstract}
The Kadanoff theory of scaling near the critical point for an Ising ferromagnet is cast in differential form. The resulting differential equations are an example of the differential equations of the renormalization group. It is shown that the Widom-Kadanoff scaling laws arise naturally from these differential equations if the coefficients in the equations are analytic at the critical point. A generalization of the Kadanoff scaling picture involving an "irrelevant" variable is considered; in this case the scaling laws result from the renormalization-group equations only if the solution of the equations goes asymptotically to a fixed point.
\end{abstract}

The problem of critical behavior in ferromagnets (and other systems) has long been a puzzle. ${ }^{1}$ Consider the Ising model of a ferromagnet; the partition function is

$$
Z(K, h)=\sum_{\{s\}} \exp \left(K \sum_{\overrightarrow{\mathbf{i}}} \sum_{i} s_{\overrightarrow{\mathbf{i}}} s_{\overrightarrow{\mathbf{i}}+\hat{i}}+h \sum_{\overrightarrow{\mathbf{i}}} s_{\overrightarrow{\mathbf{I}}}\right),
$$

where $K=-J / k T, J$ is a coupling constant, $s_{\mathbf{n}}$ is the spin at lattice site $\overrightarrow{\mathrm{n}}, \Gamma_{i}$ is a sum over nearestneighbor sites, and $h$ is a magnetic field variable. The spin $s_{\overrightarrow{\mathrm{n}}}$ is restricted to be $\pm 1 ; \sum_{\{}$(s) means a sum over all possible configurations of the spins.
$T$ is the temperature, and $k$ is Boltzmann's constant. The partition function is a sum of exponentials each of which is analytic in $K$ and $h$. Therefore one would expect the partition function itself to be analytic in $K$ and $h$. In fact, however, the partition function is singular for $K=k_{c}$ and $h=0$, where $K_{c}$ is the critical value of $K$. To be precise, the singularity occurs only in the infinite-volume limit, in which case one calculates the free-energy density

$$
F(K, h)=\lim _{y \rightarrow \infty} \frac{1}{V} \ln Z(K, h),
$$


where $V$ is the volume of the system.

Because of the infinite-volume limit there is no formal contradiction in the result that $F(K, h)$ is singular at $K=K_{c}$. The problem is that the methods one has for calculating sums such as (1) do not easily lead to singular behavior, so it is extremely difficult to get a good understanding of critical singularities working directly with the sum of Eq. (1). What one would like to do is to transform the problem of calculating $F(K, h)$ into a form where it is natural for $F$ to have singularities at $K=K_{c}$, and where one may hope the nature of the singularity will be more easily seen than from Eq. (1).

In this paper it will be suggested that an appropriate reformulation is in terms of a group, namely, the renormalization group. ${ }^{2}$ It has already been suggested that the renormalization group is important for understanding critical phenomena. ${ }^{3}$ The function of this paper is to explain what the renormalization group is and what the assumptions are that make it useful.

The renormalization group is a nonlinear transformation group of the kind that occurs in classical mechanics. The equations of motion of a classical system with time-independent potentials define transformations on phase space which form a group. The finite transformations of the group are the transformations induced by a finite translation in time; the infinitesimal transformation is defined by the equations of motion themselves. It will be shown how a translation group can arise in the analysis of critical behavior. This group is called the renormalization group for historical reasons (the connection with renormalization will be explained at the conclusion of paper $\left.\mathrm{II}^{4}\right)$. The infinitesimal transformation of the renormalization group is analogous to an equation of motion, and we shall use the language of differential equations rather than the language of group theory in the remainder of this paper.

The advantage of a reformulation of Eq. (1) in terms of the differential equations of the renormalization group is that it allows the singularities of the critical point to occur naturally. Before setting up these differential equations we shall show with a simple classical example how singularities can be generated from an equation of motion. Consider the equation

$$
\frac{d x}{d t}=-\frac{d V}{d x}(x)
$$

where $V(x)$ is the function shown in Fig. 1. One can think of this equation as describing the motion of a ball rolling on a hill with height given by $V(x)$. Equation (3) is not strictly speaking the equation of motion for said ball, but qualitatively the solution of this equation is similar to the solution of the second-order equation one should write down (this is true in particular if there are frictional forces which prevent the ball from rolling back and forth in the valleys near $x_{A}$ and $x_{B}$ of Fig. 1). Let $x_{C}$ be the location of the maximum of $V(x)$, i.e., the top of the hill. If the ball is released at any point $x>x_{C}$ (on the left of $x_{C}$ ) then the ball rolls down to the point $x_{A}$ and stops. If it is released to the right of $x_{C}$ it rolls to $x_{B}$ and stops. This means the final position of the ball is a discontinuous function of its initial location. To be precise, let the position of the ball at time $t$ be $x\left(t, x_{0}\right)$ where $x_{0}$ is the initial location (at time 0 ). Then the function $x\left(\infty, x_{0}\right)$ is a discontinuous function of $x_{0}$. There is nothing mysterious about this discontinuity, it is just that a small change in the initial condition, from $x_{0}$ slightly less than $x_{C}$ to $x_{0}$ slightly greater than $x_{c}$, can be amplified by the passage of time until for very large $t$ the difference in position is the difference in $x_{B}-x_{A}$. With an infinite length of time available one can get an infinite amount of amplification, thus leading to a discontinuity in $x\left(\infty, x_{0}\right)$ as a function of $x_{0}$ whereas $x\left(t, x_{0}\right)$ for finite $t$ is continuous. It is assumed here that the potential $V(x)$ is analytic in $x$, as indicated by Fig. 1, so the discontinuity in $x\left(\infty, x_{0}\right)$ at $x_{0}=x_{C}$ cannot be blamed on any singularity in $V(x)$ itself.

The basic proposal of this paper is that the singularities at the critical point of a ferromagnet can be understood as arising from the $t=\infty$ limit of the solution of a differential equation. In order to develop an understanding of how one relates critical behavior to a differential equation, we shall set up Kadanoff's scaling picture ${ }^{5}$ in differential form. Kadanoff's original hypothesis which led to the Widom-Kadanoff scaling laws ${ }^{1}$ was that near the critical point one could imagine blocks of spins acting as a unit, i.e., all spins in a block would be up or down simultaneously. Kadanoff then argued that one could treat all spins in a block as a single effective spin, and one could write an effective Hamiltonian in the Ising form for these effective spins. He then showed how these assumptions

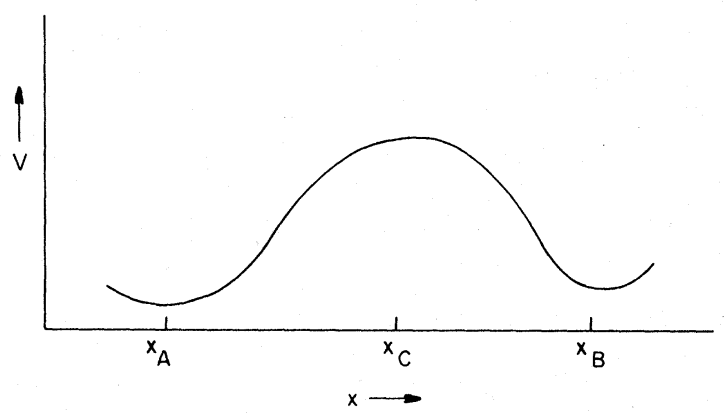

FIG. 1. Potential $V(x)$ with minima at $x_{A}$ and $x_{B}$ and a maximum at $x_{C}$. 
lead to scaling laws.

The idea that blocks of spins act as a unit near the critical temperature does not stand up to close examination; in fact only very near zero temperature $\left(K \gg K_{C}\right)$ is it true. ${ }^{5}$ The reason for discussing the Kadanoff hypothesis is that it leads to a very simple set of differential equations. By studying these differential equations, one builds an understanding of how critical singularities can emerge from a set of equations of motion. In Paper II we will discuss a generalization of the differential equations which can be more realistic than the Kadanoff block hypothesis. The intuitive picture of how the critical singularities arise from the differential equations will not be changed by the generalization, although a wider range of critical singularities is possible with the generalized equations.

In short the Kadanoff block picture, although absurd, will be the basis for generalizations which are not absurd, and it is helpful to understand the Kadanoff picture in differential form before studying these generalizations.

Imagine an infinite cubic lattice divided into cubic blocks $L$ lattice sites on a side. Each block contains $L^{3}$ lattice sites. The total spin in a block is the sum of the $L^{3}$ spins in the block. According to the Kadanoff block picture, all the spins in the block are aligned together, so the total spin has only two values, $+L^{3}$ or $-L^{3}$. The blocks of spins themselves define a lattice of spins, but the lattice spacing of the blocks is $L$ times the spacing of individual spins. Introduce a spin variable $s_{\overrightarrow{\mathrm{m}}}^{\prime}$ associated with block $\overrightarrow{\mathrm{m}}$. Normalize $s_{\overrightarrow{\mathrm{m}}}^{\prime}$ so $s_{\overrightarrow{\mathrm{m}}}^{\prime}= \pm 1$, i. e., $s_{\vec{m}}^{\prime}$ is $L^{-3} \times($ total spin in block $\overrightarrow{\mathrm{m}})$. The interactions between blocks involve only nearestneighbor blocks, and the magnetic field couples to each block separately. This suggests that the interaction energy of the blocks can be expressed in Ising form [Eq. (1)] except that one must substitute new constants $K_{L}$ and $h_{L}$ for the original parameters $K$ and $h$.

Kadanoff proposes in particular that the total free energy of the original Ising model is the same as the free energy of the blocks calculated using the block parameters. In practice this equivalence is expressed in terms of the free-energy density rather than the total free energy. Let $F(K, h)$ be the free energy per lattice site of the original Ising Hamiltonian of Eq. (1). The free energy per block of the block Hamiltonian is simply $F\left(K_{L}, h_{L}\right)$. If the total free energy is the same for both, then ${ }^{6}$

$$
F(K, h)=L^{-3} F\left(K_{L}, h_{L}\right) \text {. }
$$

In the Kadanoff picture one can also compute the correlation length using the block Hamiltonian. Let $\xi(K, h)$ be the correlation length for the original Hamiltonian in units of the lattice spacing. Then
$\xi\left(K_{L}, h_{L}\right)$ is the correlation length of the block Hamiltonian, in units of the block spacing. For the two to agree, one must have

$$
\xi(K, h)=L \xi\left(K_{L}, h_{L}\right) \text {. }
$$

The Kadanoff picture is, in summary, that there exists effective coupling parameters $K_{L}$ and $h_{L}$ such that Eqs. (4) and (5) hold, for any $L$. Kadanoff also requires that correlation functions for large distances be calculable through the block Hamiltonian, but this will not be assumed here. Kadanoff's picture requires that $L$ be an integer, but we shall assume that $L$ can be a continuous variable, in order to be able to write differential equations in $L$. Kadanoff restricts $L$ to be much less than $\xi(K, h)$; we shall allow $L$ to be arbitrary.

The differential equations of the renormalization group will be, in the Kadanoff picture, equations for $K_{L}$ and $h_{L}$. So far nothing has been said about how to compute $K_{L}$ and $h_{L}$. Kadanoff proposed definite forms for the dependence of $K_{L}$ and $h_{L}$ on $L$, namely,

$$
\begin{aligned}
& K_{L}=K_{c}-\epsilon L^{y}, \\
& h_{L}=h L^{x},
\end{aligned}
$$

where ${ }^{7}$

$$
\epsilon=K_{c}-K
$$

and Eqs. (6) and (7) are valid only for $L<<\xi(K, h)$. Here we shall first derive differential equations for $K_{L}$ and $h_{L}$ and show later that the solution of the differential equations has Kadanoff's form.

To obtain the general form of the differential equations for $K_{L}$ and $h_{L}$, we note the following. The constants $K_{2 L}$ and $h_{2 L}$ are functions of $K_{L}$ and $h_{L}$ but not of $L$ separately. The change from $K_{L}$ and $h_{L}$ to $K_{2 L}$ and $h_{2 L}$ is equivalent to making new blocks of size $2 L$ out of old blocks of size $L$. Each new block is a cube containing eight old blocks. But in writing an effective Hamiltonian with constants $K_{L}$ and $h_{L}$, one has substituted a lattice for the old blocks; having made this substitution the Hamiltonian does not know what the size $L$ of the old blocks was. Regardless of the value of $L$, the change to $2 L$ is simply a matter of combining eight lattice sites to make the new block, so $K_{2 L}$ and $h_{2 L}$ must be the same function of $K_{L}$ and $h_{L}$ for any $L$.

This continues to be true if one goes from $L$ to $3 L, L$ to $4 L$, etc. Generalizing to the continuous case, we assume this is true also for going from $L$ to $(1+\delta) L$, for small $\delta$. This means $\delta L d K_{L} / d L$ can depend on $K_{L}$ and $h_{L}$ but not $L$ separately:

$$
\frac{d K_{L}}{d L}=\frac{1}{L} u\left(K_{L}, h_{L}{ }^{2}\right)
$$

We expect $u$ to depend only on $h_{L}^{2}$ owing to the symmetry of the Ising Hamiltonian for ${ }^{8} h_{L} \rightarrow-h_{L}$. The 
analogous equation for $h_{L}$ is

$$
\frac{d h_{L}}{d L}=L^{-1} h_{L} v\left(K_{L}, h_{L}^{2}\right)
$$

Equations (9) and (10) are the renormalizationgroup equations suggested by the Kadanoff block picture. Because of the questionable validity of this picture one would expect the differential equations to be equally questionable. Actually this is not so; there is another way to derive the differential equations which involves only minimal assumptions, such that the differential equations become essentially a tautology. Namely, let us define $K_{L}$ and $h_{L}$ to be the solutions of Eqs. (4) and (5). That is, we assume that one has found the exact solution of the Ising model, as a function of $K$ and $h$, and regard Eqs. (4) and (5) as giving implicit definitions of $K_{L}$ and $h_{L}$ for any $L$. Assume that these equations have a unique solution for any $L$. The differential equations are now trivial to obtain: Differentiate both Eqs. (4) and (5) with respect to $L$. One gets

$$
\begin{aligned}
& 0=L^{-3}\left(-\frac{3}{L} F\left(K_{L}, h_{L}\right)+\frac{d K_{L}}{d L} \frac{\partial F\left(K_{L}, h_{L}\right)}{\partial K_{L}}\right. \\
& \left.+\frac{d h_{L}}{d L} \quad \frac{\partial F\left(K_{L}, h_{L}\right)}{\partial h_{L}}\right) \\
& 0=L\left(\frac{\xi\left(K_{L}, h_{L}\right)}{L}+\frac{d K_{L}}{d L} \frac{\partial \xi\left(K_{L}, h_{L}\right)}{\partial K_{L}}+\frac{d h_{L}}{d L} \frac{\partial \xi\left(K_{L}, h_{L}\right)}{\partial h_{L}}\right) .
\end{aligned}
$$

These equations can be solved for $d K_{L} / d L$ and $d h_{L} / d L$, to give Eqs. (9) and (10), with

$$
\begin{aligned}
u\left(K_{L}, h_{L}^{2}\right)= & \left(3 F\left(K_{L}, h_{L}\right) \frac{\partial \xi}{\partial h_{L}}\left(K_{L}, h_{L}\right)\right. \\
& \left.+\xi\left(K_{L}, h_{L}\right) \frac{\partial F}{\partial h_{L}}\left(K_{L}, h_{L}\right)\right) \\
& \times\left(\frac{\partial F}{\partial K_{L}}\left(K_{L}, h_{L}\right) \frac{\partial \xi}{\partial h_{L}}\left(K_{L}, h_{L}\right)\right. \\
& \left.-\frac{\partial \xi}{\partial K_{L}}\left(K_{L}, h_{L}\right) \frac{\partial F}{\partial h_{L}}\left(K_{L}, h_{L}\right)\right)^{-1}
\end{aligned}
$$

and a similar formula for $v\left(K_{L}, h_{L}^{2}\right) .^{9}$ Note that $u\left(K_{L}, h_{L}^{2}\right)$ does not depend on $L$ (except through $K_{L}$ and $h_{L}$ ), as expected. This result means that one has an explicit formula for the function $u\left(K, h^{2}\right)$ in terms of $F(K, h)$ and $\xi(K, h)$ and their derivatives.

The differential equations thus obtained may not be very interesting. The reason is this. The idea of converting the Ising problem into a group was that the singularities at the critical point should result from solving the group equations. This makes sense only if the differential equations are themselves free of singularities at $K_{c}$. Referring back to the analogy of a ball on the hill, the function $V(x)$ can be analytic in $x$ and still have $x\left(\infty, x_{0}\right)$ be discontinuous in $x_{0}$. Analogously we would like $u\left(K, h^{2}\right)$ and $v\left(K, h^{2}\right)$ to be analytic at the critical point. The trouble with Eq. (13) is that it expresses $u\left(K, h^{2}\right)$ in terms of $F(K, h)$ and $\xi(K, h)$, both of which are singular at the critical point, implying that $u$ is singular also. ${ }^{10}$ Most previous formulations of the renormalization group used equations such as Eq. (13) to define the functions $u$ and $v$ which appear in the renormalization-group equations. This has been the cause of much confusion about the purpose of the renormalization group.

The Kadanoff block hypothesis suggests that $u\left(K, h^{2}\right)$ and $v\left(K, h^{2}\right)$ will indeed be analytic at the critical point. In the block picture one should be able to construct $K_{L}$ and $h_{L}$ just by adding up interactions of individual spins within a block or across the boundary between two blocks; it is hard to see how this simple addition over a finite region can lead to singular expressions for $K_{L}$ and $h_{L}$ as a function of $K$ and $h$, if $L$ is fixed. More generally this process should give $K_{n L}$ and $h_{n L}$ as analytic functions of $K_{L}$ and $h_{L}$ for fixed $n$; specializing to $n=1$ $+\delta$, the functions $u$ and $v$ should be analytic. However, in the spirit of the Kadanoff approach one does not try to get specific forms for $u\left(K, h^{2}\right)$ and $v\left(K, h^{2}\right)$ because this would require that one take literally the idea that all spins within a block act as a unit. An explicit realization of the renormalization-group equations will be presented in Paper II. ${ }^{4}$

Another feature of the renormalization-group equations suggested by the Kadanoff block picture is the following: In classical physics it is usually easier to write down the equation of motion, such as Eq. (3), than to write down the solution of the equation. Typically the potential $V(x)$ is a simple function, or easily approximated by a simple function. The solution of the equation can be much more complicated, especially if one has coupled differential equations to solve. The Kadanoff block hypothesis suggests that the renormalizationgroup differential equations will also be simpler than their solution. This is because the smaller $L$ is, the fewer the interactions that have to be summed to give $K_{L}$ and $h_{L}$. It is not immediately obvious that $L=1+\delta$ is easier to compute than $L=2$, but this is not the point. The point is that $L=2,3$, or 4 is much easier to compute than $L=10000$; one expects $L=1+\delta$ also to be easier than $L=10000$.

If it is true that the renormalization-group equation is easier to write down than its solution, then it is natural to try to solve the Ising model by first obtaining the renormalization-group equations and then trying to integrate them. Specializing to the problem of critical behavior, what one thinks of do- 
ing is integrating the equations until $L$ is of order $\xi$. When $L$ is of order $\xi, \xi\left(K_{L}, h_{L}\right)$ is of order 1 [by Eq. (5)], which means $K_{L}$ and $h_{L}$ must be well away from their critical values; then it is easy to compute $\xi\left(K_{L}, h_{L}\right)$ and $F\left(K_{L}, h_{L}\right)$ by other means and reconstruct $F(K, h)$ and $\xi(K, h)$ from Eqs. (4) and (5). This integration procedure can be carried out for $K$ and $h$ near their critical values, and if $u(K, h)$ and $v(K, h)$ are analytic at the critical point, one obtains immediately the Widom-Kadanoff scaling laws.

To make the calculation precise, let us proceed as follows. Imagine that one is at a temperature slightly above $T_{c}$. This means $K$ is slightly smaller than $K_{c}$. As $L$ increases $K_{L}$ must decrease, so as to go away from $K_{c}$. This ensures that $\xi\left(K_{L}, h_{L}\right)$ decreases as $L$ increases, as required by Eq. (5). Pick a value for $K_{L}$ well away from $K_{c}$, say $K_{c} / 2$; let us integrate the renormalization-group equations until a value of $L$ is reached for which $K_{L}=K_{c} / 2$, then stop and compute $F(K, h)$ and $\xi(K, h)$ from Eqs. (4) and (5).

If $K$ and $h$ have exactly the critical, values $K_{c}$ and 0 , respectively, then one must have $K_{L}=K_{c}$ and $h_{L}=0$ for all $L$. The reason for this is that $\xi\left(K_{c}, 0\right)$ is infinite; therefore $\xi\left(K_{L}, h_{L}\right)$ must be infinite for all $L$. For $\xi\left(K_{L}, h_{L}\right)$ to be infinite, $K_{L}$ and $h_{L}$ must have the critical values. Hence $K_{L} \equiv K_{c}$ and $h_{L} \equiv 0$ must be a solution of the renormalization group equations, which is true only if

$$
u\left(K_{c}, 0\right)=0 \text {. }
$$

Comparing this result to the classical analog of the ball on the hill, the point $K=K_{c}$ is analogous to one of the points of equilibrium for the ball $\left(x=x_{A}\right.$, or $x_{B}$, or $\left.x_{C}\right)$.

Now let $K$ and $h$ be near the critical values $K_{c}$ and 0 . For small values of $L$, namely for $L$ $\ll \xi(K, h)$, the effective correlation length $\xi\left(K_{L}, h_{L}\right)$ will be large and $K_{L}$ and $h_{L}$ will also be near the critical values. For this range of $L$ one can use a linearized form of the renormalization group equations to compute $K_{L}$ and $h_{L}$. The linearized equations for $K_{L}$ and $h_{L}$ are

$$
\begin{aligned}
& \frac{d K_{L}}{d L} \simeq \frac{1}{L}\left(K_{L}-K_{c}\right) y, \\
& \frac{d h_{L}}{d L} \simeq \frac{1}{L} h_{L} x,
\end{aligned}
$$

where $x$ and $y$ are constants:

$$
\begin{aligned}
& y=\frac{\partial u}{\partial K}\left(K_{c}, 0\right), \\
& x=v\left(K_{c}, 0\right) .
\end{aligned}
$$

In writing these equations we have assumed that $u$ and $v$ are differentiable at the critical point; this is how one uses in practice the analyticity predicted from the Kadanoff block picture. Using the linearized equations of the renormalization group is analogous to replacing $V(x)$ by a quadratic form when $x$ is near $x_{C}$.

The solutions of Eqs. (15) and (16) are the formulas (6) and (7) proposed by Kadanoff. Assume that this approximation is valid until $K_{L}=K_{c} / 2$. Then one can solve for the value of $L$ giving $K_{L}$ $=K_{c} / 2$ :

$$
K_{c} / 2=\epsilon L^{y}
$$

so

$$
L=\left(K_{c} / 2 \epsilon\right)^{1 / y},
$$

which is a scaling law for $L$ as a function of $\epsilon$. For this value of $L, h_{L}$ is

$$
h_{L}=h\left(K_{c} / 2 \epsilon\right)^{x / y} .
$$

Hence one can compute $F(K, h)$ and $\xi(K, h)$ to be

$$
\begin{gathered}
F(K, h)=\left(K_{c} / 2 \epsilon\right)^{-3 / y} F\left[K_{c} / 2, h\left(K_{c} / 2 \epsilon\right)^{x / y}\right], \\
\xi(K, h)=\left(K_{c} / 2 \epsilon\right)^{1 / y} \xi\left[K_{c} / 2, h\left(K_{c} / 2 \epsilon\right)^{x / y}\right] .
\end{gathered}
$$

These formulas are scaling laws; the functions $F(K, h)$ and $\xi(K, h)$ depending on two variables have been reduced to explicit powers of $\epsilon$ (i.e., $T-T_{c}$ ) multiplying functions depending only on the single variable $h \epsilon^{-x / y}$. For consequences of these laws see Ref. 1.

To be accurate we should have integrated the nonlinear equations once $K_{L}-K_{c}$ became large. What can one say about the solution $K_{L}$ and $h_{L}$ in this case? One can say the following: Let

$$
\begin{gathered}
K_{L}=\phi\left(L, h_{0}\right), \\
h_{L}=\psi\left(L, h_{0}\right)
\end{gathered}
$$

be the solution of the exact equations (9) and (10) over the range $0<L<\infty$ satisfying the boundary conditions

$$
\begin{aligned}
& \phi\left(1, h_{0}\right)=K_{c} / 2, \\
& \psi\left(1, h_{0}\right)=h_{0},
\end{aligned}
$$

that is, boundary conditions well away from the critical point. For $L \ll 1$ this solution should be near the critical point. Hence for $L \ll 1, K_{L}$ and $h_{L}$ should be solutions of the linearized equations, giving

$$
\begin{aligned}
& \phi\left(L, h_{0}\right) \approx \phi\left(h_{0}\right) L^{y}+K_{c}, \\
& \psi\left(L, h_{0}\right) \approx \psi\left(h_{0}\right) L^{x}
\end{aligned}
$$

for $L \ll 1$ where $\phi\left(h_{0}\right)$ and $\psi\left(h_{0}\right)$ are constants depending on $h_{0}$. Now the exact renormalization-group equations have translational symmetry when written in terms of $\ln L$, just as the classical equation (3) does. Hence the functions

$$
K_{L}=\phi\left(a L, h_{0}\right),
$$




$$
h_{L}=\psi\left(a L, h_{0}\right)
$$

are solutions of the renormalization-group equations where $a$ is any constant. For $a L \ll 1$ this solution reduces to

$$
\begin{aligned}
K_{L} & =\phi\left(h_{0}\right)(a L)^{y}+K_{c}, \\
h_{L} & =\psi\left(h_{0}\right)(a L)^{x} .
\end{aligned}
$$

This solution can be matched to any given initial condition for $K$ and $h$; if $K$ and $h$ are near their critical values then $K$ and $h$ can be matched to the asymptotic forms (32) and (33), giving

$$
\begin{aligned}
& \epsilon=a^{y} \phi\left(h_{0}\right), \\
& h=a^{x} \psi\left(h_{0}\right) .
\end{aligned}
$$

This means that

$$
h \epsilon^{-x / y}=\psi\left(h_{0}\right) /\left[\phi\left(h_{0}\right)\right]^{1 / y},
$$

which is an implicit equation for $h_{0} ; h_{0}$ depends only on the single variable $h \epsilon^{-x / y}$. Then, from Eq. (34), we have

$$
a=\left[\epsilon / \phi\left(h_{0}\right)\right]^{1 / y} .
$$

But now the value of $L$ for which $K_{L}=K_{c} / 2$ is $L$ $=a^{-1}$, from the boundary condition on $\phi[\mathrm{Eq}$. (26)]; and for this value of $L, h_{L}=h_{0}[\mathrm{Eq} .(27)]$. Using Eqs. (4) and (5) one again gets scaling laws for $F$ and $\xi$, for example

$$
\xi(K, h)=\left[\epsilon / \phi\left(h_{0}\right)\right]^{-1 / y} \xi\left(K_{c} / 2, h_{0}\right),
$$

where $h_{0}$ depends only on $h \epsilon^{-x / y}$. This scaling law is the same as (23) except for a different functional dependence on the scaling variable $h \epsilon^{-x / y}$.

These results can be related to the classical analogue of the ball on a hill. Consider only the case of no magnetic field $(h=0)$ in which case $h_{L}=0$ for all $L$ and one has left a single dependent variable $K_{L}$. With $h=0, h_{0}$ is zero also; $\phi(0)$ is a constant $\phi_{0}$ and the formula for $\xi(K, 0)$ is

$$
\xi(K, 0)=\left(\epsilon / \phi_{0}\right)^{1 / y} \xi_{0},
$$

where $\xi_{0}$ is $\xi\left(\frac{1}{2} K_{c}, 0\right)$.

The point $K=K_{c}$ is analogous to the top of the hill $\left(x=x_{C}\right)$. This is because if $K$ is different from $K_{c}$ then $K_{L}-K_{c}$ increases as $L$ increases, and this is analogous to the ball rolling away from the top of the hill. In other words the point $K=K_{c}$ is analogous to a point of unstable equilibrium in classical mechanics. What is the analogue of the correlation length $\xi(K, 0)$ ? From Eq. (5), $\xi(K, 0)$ is.proportional to $L$ where $L$ is chosen to make $K_{L}=K_{c} / 2$. This is analogous to computing the time $t_{1 / 2}$ for the ball to roll half-way down the hill. As the initial location $x_{0}$ of the ball approaches the top of the hill, this time $t_{1 / 2}$ increases, becoming infinite as $x_{0}$ $\rightarrow x_{C}$. The scaling law for $\xi$ is obtained by computing the dependence of $L$ on $K-K_{c}$ for $K$ near $K_{c}$. This is analogous to finding the dependence of $t_{1 / 2}$ on $x_{0}-x_{c}$. Most of the time $t_{1 / 2}$ is spent near the top of the hill, and so the dependence of $t_{1 / 2}$ on $x_{0}-x_{C}$ can be determined to a good approximation from linearized equations about the unstable equilibrium point $x_{C}$.

This completes the discussion of the simplest renormalization-group equations following from the Kadanoff block picture. One sees from this how the idea of differential equations with analytic coefficients leads to singularities at the critical point satisfying the Widom-Kadanoff scaling laws. The critical point corresponds to an unstable equilibrium point of the differential equation, and the singularity at the critical point is a consequence of the infinite time required to move away from a point of unstable equilibrium.

The problem with the simple renormalizationgroup equations discussed earlier is that there is at present no hope of showing that the functions $u\left(K, h^{2}\right)$ and $v\left(K, h^{2}\right)$ are analytic at the critical point. Without the analyticity, the renormalization-group equations become a tautology, as explained earlier. Instead of trying to prove that $u$ and $v$ are analytic, one can try to generalize the renormalization-group equations in the hope that analyticity will be easier to establish for the generalization. The generalizations which the author has been able to construct are rather complicated, involving an infinite number of $L$-dependent coupling constants. To prepare for these generalizations it is worth discussing the nature of the renormalization-group equations when they involve one additional coupling constant $q_{L}$. The initial variable $q$ will be an "irrelevant" variable in Kadanoff's language. ${ }^{11}$ It might be the coefficient of a secondnearest-neighbor coupling, for example. The variable $q$ will prove to be irrelevant only in certain respects that will be explained later.

Imagine that the renormalization-group equations involve three $L$-dependent variables $K_{L}, q_{L}$, and $h_{L}$. We think of $K_{L}$ and $q_{L}$ as coefficients of firstand second-nearest-neighbor couplings which are even to the exchange $s_{\overrightarrow{\mathrm{m}}}^{\prime} \rightarrow-s_{\mathrm{m}}^{\prime}$ while $h_{L}$ still multiplies the spins $s_{\overrightarrow{\mathrm{m}}}^{\prime}$ themselves which are odd to this exchange. Hence one expects the form of the renormalization-group equations to be

$$
\begin{aligned}
& \frac{d K_{L}}{d L}=\frac{1}{L} u\left(K_{L}, q_{L}, h_{L}^{2}\right), \\
& \frac{d q_{L}}{d L}=\frac{1}{L} w\left(K_{L}, q_{L}, h_{L}^{2}\right), \\
& \frac{d h_{L}}{d L}=\frac{1}{L} h_{L} v\left(K_{L}, q_{L}, h_{L}^{2}\right) .
\end{aligned}
$$

The initial values of $K_{L}, q_{L}$, and $h_{L}$ (for $L=1$ ) are denoted $K, q$, and $h$. We assume the same rules for computing the free energy and the correlation 
length as before, namely,

$$
\begin{gathered}
F(K, q, h)=L^{-3} F\left(K_{L}, q_{L}, h_{L}\right), \\
\xi(K, q, h)=L \xi\left(K_{L}, q_{L}, h_{L}\right) .
\end{gathered}
$$

We now expect there will be a line of critical points; for each value of $q$ there should be a critical value $K_{c}(q)$ for $K$. If the initial values of $K$, $q$, and $h$ lie on the critical line $\left(K=K_{c}(q), h=0\right)$ then the solution $K_{L}, q_{L}, h_{L}$ must lie on the critical line for all $L$, in order that $\xi\left(K_{L}, q_{L}, h_{L}\right)$ be infinite for all $L$. Hence, if $K=K_{c}(q)$ and $h=0$ one can write $K_{L}=K_{C}\left(q_{L}\right)$ and $h_{L}=0$; the equation for $q_{L}$ becomes

$$
\frac{d q_{L}}{d L}=\frac{1}{L} w_{c}\left(q_{L}\right)
$$

where

$$
w_{c}\left(q_{L}\right)=w\left(K_{c}\left(q_{L}\right), q_{L}, 0\right) .
$$

This is a one-dimensional equation of motion, which is directly analogous to the classical equation of motion for the ball on a hill discussed earlier. In the limit $L \rightarrow \infty$ there is the possibility that $q_{L}$ approaches an equilibrium point of the differential equation. This is not the only possibility; it is also possible that $q_{L} \rightarrow \infty$ as $L \rightarrow \infty$. We shall study only the case that $q_{L}$ approaches as equilibrium point, this being the case that is easiest to study. This means we will not give a completely general discussion of the solution of the renormalizationgroup equations. In fact a general discussion is very complicated and becomes hopeless when the equations are generalized further.

Let the equilibrium point approached by $q_{L}$ be $q_{c}$, and let $K_{c}\left(q_{c}\right)$ be $K_{c}$. If one chooses $q=q_{c}$ and $K=K_{c}$, then $q_{L} \equiv q_{c}$ for all $L, K \equiv K_{c}$ for all $L$ so one has an equilibrium point of the full renormalization-group equations.

Let us study the solutions of the renormalizationgroup equations in linearized form about the equilibrium point $K_{c}, q_{c}$. The linearized equations are

$$
\begin{aligned}
& L \frac{d K_{L}}{d L}=y_{11}\left(K_{L}-K_{c}\right)+y_{12}\left(q_{L}-q_{c}\right), \\
& L \frac{d q_{L}}{d L}=y_{21}\left(K_{L}-K_{c}\right)+y_{22}\left(q_{L}-q_{c}\right), \\
& L \frac{d h_{L}}{d L}=x h_{L},
\end{aligned}
$$

where

$$
\begin{aligned}
& \mathrm{y}_{11}=\frac{\partial \mathrm{u}}{\partial K}\left(K_{c}, q_{c}, 0\right), \\
& \mathrm{y}_{12}=\frac{\partial \mathrm{u}}{\partial q}\left(K_{c}, q_{c}, 0\right), \\
& \mathrm{y}_{21}=\frac{\partial w}{\partial K}\left(K_{c}, q_{c}, 0\right),
\end{aligned}
$$

$$
\begin{aligned}
& \mathrm{y}_{22}=\frac{\partial w}{\partial q}\left(K_{c}, q_{c}, 0\right), \\
& \text { and } \\
& x=v\left(K_{c}, q_{c}, 0\right) .
\end{aligned}
$$

There will, in general, be two linearly independent solutions of the first two equations behaving as a power of $L$, say ${ }^{12}$

$$
K_{L}-K_{c}=L^{y}, q_{L}-q_{c}=r_{y} L^{y}
$$

and

$$
K_{L}-K_{c}=L^{z}, \quad q_{L}-q_{c}=r_{z} L^{Z} .
$$

One of these solutions must be decreasing as $L \rightarrow \infty$, since if the initial value of $K$ is $K_{c}(q)$ then $K_{L} \rightarrow K_{c}$ and $q_{L} \rightarrow q_{c}$ as $L \rightarrow \infty$, and this is not possible if both $y$ and $z$ are positive. Let $z$ therefore be the negative exponent. The exponent $y$ must be positive so that $K_{L}$ goes away from $K_{c}$ if $K \neq K_{c}(q)$. What this means is that the point $K_{c}, q_{c}$ must be analogous to a saddle point in classical mechanics which is stable from one direction but unstable in the orthogonal direction.

One can set up a classical analog to the coupled equations for $K_{L}$ and $q_{L}$. Consider only the case $h_{L} \equiv 0$, for simplicity. A classical analog is the pair of equations

$$
\begin{aligned}
& \frac{d K}{d t}(t)=-\frac{\partial V}{\partial K}(K, q), \\
& \frac{d q}{d t}(t)=-\frac{\partial V}{\partial q}(K, q) .
\end{aligned}
$$

This is not as general as Eqs. (40) and (41), since it is not true in general that two functions $u$ and $w$ can be written as the gradient of a potential. But we can illustrate the idea that $K_{c}, q_{c}$ define a saddle point with this example. Think of Eqs. (57) and (58) as describing the motion of a ball on a twodimensional terrain, with $V(K, q)$ being the elevation at the point $(K, q)$. It is convenient to illustrate $V(K, q)$ by a contour map, as in Fig. 2. Figure 2 shows a depression at the point $P_{A}$ (the origin); $P_{B}$ is the top of a hill and $P_{C}$ a saddle point. There is a ridge going down the hill to the saddle and a gully from the saddle to the bottom of the depression. $q_{0}$ is an arbitrary value of $q$. If one starts the ball at coordinates $q_{0}$ and a small value of $K$, it will simply roll to the bottom of the depression. If one increases the initial value of $K$, the ball will still roll to $P_{A}$, until one starts at the point $P_{D}$ exactly on the ridge. In this case the ball rolls to the saddle point $P_{C}$ and stops. If one starts just short of $P_{D}$, the ball rolls almost to $P_{C}$ and then goes down the gully to $P_{A}$. The ball moves very slowly when it is near $P_{C}$ and a large time elapses before it moves an appreciable distance down the gully. 


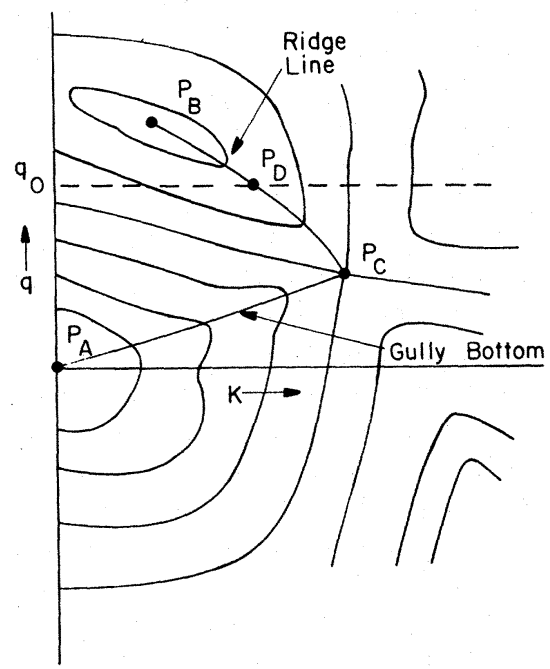

FIG. 2. Potential $V(K, q)$ plotted by contours of constant $V$. The minimum of $V$ is at the origin $\left(P_{A}\right)$; the maximum is at $P_{B}$ and $P_{C}$ is a saddle point. The ridge line is the trajectory going to $P_{C}$; the gully line is the trajectory from $P_{C}$ to $P_{A}$. Unmarked lines are contours. The dashed line marks an arbitrary value $\left(q_{0}\right)$ for $q$.

The ridge line is analogous to the critical line $K=K_{c}(q)$ and the saddle point $P_{c}$ is analogous to the point $K_{c}, q_{c}$. Note that the initial location can be anywhere along the ridge line, and still the ball will roll down to the saddle point $P_{c}$, or if one starts just short of the ridge line, the path of the ball will just miss $P_{C}$ and go down the gully line, independently of where along the ridge line one started. It is in this sense that $q$ is an irrelevant variable. To be fairer, there is one relevant and one irrelevant variable in the pair $(K, q)$ but whether $K$ or $q$ is called the irrelevant variable does not matter. If one looks at the functions $K_{L}, q_{L}$ starting from an initial point close to the critical line, then as $L$ increases $\left(K_{L}, q_{L}\right)$ approaches the saddle point $\left(K_{c}, q_{c}\right)$. By $L=5$ or 10 one should be close to $K_{c}, q_{c}$ (unless $z \simeq 0$ so the rate of approach is slow). Then $K_{L}$ and $q_{L}$ change very little until $L$ becomes of order of the correlation length $\xi(K, q)$, at which point $K_{L}$ and $q_{L}$ move away from the saddle point. In the classical analog, the large $L$ part of the curve $K_{L}, q_{L}$ is the gully line, and this is independent of the irrelevant variable. Also the value of $L$ at which $K_{L}=K_{c} / 2$ should be essentially independent of where along the ridge line one starts; how large $L$ is is determined by how far from the ridge line one starts.

Returning to the solution of the linearized equations, the general solution is a linear combination of the three simple power solutions, namely,

$$
K_{L}=K_{c}-\epsilon L^{y}+\eta L^{z}
$$

$$
\begin{aligned}
& q_{L}=q_{c}-\epsilon r_{y} L^{y}+\eta r_{z} L^{z}, \\
& h_{L}=h L^{x},
\end{aligned}
$$

where $\epsilon$ and $\eta$ depend on the initial values $K$ and $q$ :

$$
\begin{gathered}
\epsilon=\frac{r_{z}\left(K-K_{c}\right)-\left(q-q_{c}\right)}{r_{y}-r_{z}}, \\
\eta=\frac{r_{y}\left(K-K_{c}\right)-\left(q-q_{c}\right)}{r_{y}-r_{z}} .
\end{gathered}
$$

One is on the critical line if $\epsilon=h=0$ and $\eta \neq 0$, for then $K_{L} \rightarrow K_{c}, q_{L} \rightarrow q_{c}$ when $L \rightarrow \infty$. One is near the critical line away from $K_{c}$ and $q_{c}$ if $\epsilon \ll \eta$ and $h$ is small. Then for $L \sim 1$, the $\eta$ term dominates, and since $z$ is negative, $K_{L}$ and $q_{L}$ approach $K_{c}$ and $q_{c}$ (i.e., the ball is rolling down the ridge to the saddle point). For large enough $L$ the $\epsilon$ term dominates and $K_{L}$ and $q_{L}$ move away from $K_{C}$ and $q_{c}$. The $\eta$ term continues to decrease and can be neglected. Since $\eta$ measures the initial location along the critical line, independence of $\eta$ is independence of the initial location on the critical line. One can now compute the value of $L$ for which $K_{L}=K_{c} / 2$, giving

$$
K_{c} / 2 \simeq \epsilon L^{y}
$$

or

$$
L=\left(K_{c} / 2 \epsilon\right)^{1 / y}
$$

as before. For this value of $L$,

$$
\begin{aligned}
& q_{L}=q_{c}-r_{y}\left(K_{c} / 2\right), \\
& h_{L}=h\left(K_{c} / 2 \epsilon\right)^{x / y} .
\end{aligned}
$$

The important property of these formulas is that $q_{L}$ is a constant independent of $h, \epsilon$, or $\eta$, while $h_{L}$ again depends only on the ratio $h \epsilon^{-x / y}$. So from Eqs. (43) and (44) one gets the same form of scaling laws for $F(K, h, q)$ and $\xi(K, h, q)$ as was obtained earlier when there was no irrelevant variable $q$. Since $K=K_{c}(q)$ for $\epsilon=0$, it follows from Eq. (62) that $\epsilon$ is proportional to $K-K_{c}(q)$, which is proportional to $T-T_{c}(q)$. Hence no matter what $q$ is, $\epsilon$ is the customary temperature variable $T-T_{c}$ apart from a meaningless normalization factor.

So far nothing has been said about initial conditions below $T_{c}$, i. e., $K>K_{c}(q)$. This is easily handled by the same analysis except that when $K_{L}$ moves away from $K_{c}$ it increases instead of decreasing. Hence one cannot compute the value of $L$ for which $K_{L}=K_{c} / 2$. Instead one can find the value of $L$ for which $K_{L}=2 K_{c}$, say. In the classical anlaog, when $K>K_{c}(q)$ the ball rolls down the opposite side of the hill from the gully, and one measures the time required to reach the line $K=2 K_{c}$ instead of the time to reach $K_{c} / 2$. It must be noted, however, that for $K>K_{c}(q)$ and $h=0$ one is on the boundary between the two low-temperature 
phases (the two phases corresponding to positive or negative magnetization). As $L$ increases $K_{L}$ moves away from the critical value $K_{c}(q)$, but $h_{L}$ is still zero so one is still on the boundary between the two phases. So for the renormaliztion group to be useful below $T_{c}$ one must be able to calculate the free energy and correlation length by other methods on the phase boundary well below $T_{c}$.

It was important in the analysis that $z$ be negative. If $z$ were positive one would have a point like $P_{B}$ unstable in all directions instead of a saddle point. The only way to reach $P_{B}$ is to sit on it to start with which means fixing both $K$ and $q$. In general a fixed point with both $y$ and $z$ as well as $x$ positive is still a critical point but one which requires fixing three thermodynamic quantities instead of two. ${ }^{13}$ An exceptional circumstance arises when $z=0$; the analysis of this case is complicated but still feasible. The principal results are that the critical point may or may not require fixing a third thermodynamic variable, and there can be logarithmic violations of the scaling laws.

The above calculations used the linearized form of the renormalization-group equations, but the conclusions will not be changed by using the nonlinear equations.

To summarize the results of this analysis, one starts with values of $K$ and $q$ near the critical line $K=K_{c}(q)$. The functions $K_{L}$ and $q_{L}$ have an initial transient behavior in which $K_{L}$ and $q_{L}$ adjust to the critical values $K_{c}$ and $q_{c}$. In other words, while the initial Ising Hamiltonian can have an arbitrary fraction of second-nearest-neighbor coupling, by the time one has gone to a block Hamiltonian with reasonably large block size the couplings have become essentially fixed at $K_{c}$ and $q_{c}$. The Hamiltonians for larger $L$ are therefore independent of the initial fraction of second-nearest-neighbor coupling, and the critical behavior of the theory is likewise independent of the initial fraction.

In fact the parameters of the critical behavior such as the exponents $x$ and $y$ are determined by the renormalization-group differential equation, rather than the initial Hamiltonian. If one knows the form of the functions $u, v$, and $w$, one can calculate the exponents $x$ and $y$ by finding the saddle point $K_{c}, q_{c}$ of the differential equation and then solving the linearized equations about the saddle point. In principle one then has two choices for how to find the exponents $x$ and $y$, one choice being as just described, the other choice being to compute the partition function directly and extract the critical exponents. However there is a difference in the two approaches in that the first choice (working from the differential equation) is a well-posed problem, while solving for the partition function is not. Solving for a saddle point of the differential equation is a well-posed problem in the sense that small modifications of the functions $u, v$, and $w$ make only small changes in $K_{c}, q_{c}$, and $x$ and $y$; in other words one can get approximate values of $K_{c}$ and $q_{c}$ by requiring that $u$ and $w$ only vanish approximately. In contrast, to get the singular part of the free energy requires a calculation to infinite precision since the singular part approaches zero as $K \rightarrow K_{c}(q)$ and one must know how it approaches zero; the singular term exists underneath a finite nonsingular term which is why infinite precision is required.

Clearly, if the renormalization-group picture of critical behavior is correct, it is important to derive the differential equation of the renormalization group and try to find the critical saddle point of the equation. Extrapolating from the analysis of this paper, it is clear that the renormalizationgroup equations can involve any number of coupling constants, not just two or three; one will still get the Widom-Kadanoff scaling laws provided the solution of the equations approaches a saddle point when one is at the critical temperature. If there are $n$ coupling constants instead of three, the only change will be that there will be $n-2$ linearly independent initial transient solutions of the linearized equations, instead of one, and hence $n-2$ irrelevant variables. However the equations with $n$ variables are complicated, if $n$ is large, and it is not at all certain that critical behavior would be caused by a saddle point. The solutions of the renormalizationgroup equations might instead approach a limit cycle $^{14}$ or go off to infinity or go into irregular oscillations (ergodic or turbulent?) as $L \rightarrow \infty$. So while generalized renormalization-group equations are capable of reproducing the Widom-Kadanoff theory, they are also capable of producing more challenging types of behavior.

I have benefitted from many discussions with Professor M. Fisher, Professor B. Widom, Professor L. Kadanoff, Professor D. Jasnow, Professor M. Wortis, and many others in the field of critical phenomena.

\footnotetext{
*Supported by the National Science Foundation.

${ }^{1}$ For reviews of the theory of critical phenomena, see M. Fisher, Rept. Progr. Phys. 30, 731 (1967); L. P. Kadanoff et al., Rev. Mod. Phys. 39, 395 (1967).

${ }^{2}$ The original references on the renormalization group are M. Gell-Mann and F. E. Low, Phys. Rev. 95, 1300 (1954); E. C. G. Stueckelberg and A. Petermann, Helv.
}

Phys. Acta 26, 499 (1953); N. N. Bogoliubov and D. V. Shirkov, Introduction to the Theory of Quantized Fields (Interscience, New York, 1959), Chap. VIII. For a recent review see K. Wilson, Phys. Rev. D 3 , 1818 (1971).

${ }^{3}$ C. DiCastro and G. Jona-Lasinio, Phys. Letters $29 \mathrm{~A}$, 322 (1969). Renormalization-group ideas are also used in the program of Migdal and others: See A. A. Migdal, 
Zh. Eksperim. i Teor. Fiz. $\underline{59}, 1015$ (1970) [Sov. Phys. JETP $32,552(1971)]$, and references cited therein.

${ }^{4} \mathrm{~K}$. Wilson, following paper, Phys. Rev. B $\underline{4}, 3184$ (1971), referred to as II.

${ }^{5}$ L. P. Kadanoff, Physics $\underline{2}, 263$ (1966); and L. P. Kadanoff et al. (Ref. 1). Kadanoff assumed that a block of spins of size $L$ would have a magnetization $\pm L^{d-\psi}$ with $\psi$ unknown. In this paper there is no need to use this precise form of Kadanoff's hypothesis, so for simplicity $\psi$ will be set equal to zero. The choice $\psi=0$ is false, as explained here; but even with $\psi$ an undetermined parameter no one has been able to justify Kadanoff's idea that a block of spins acts as a unit with only two possible values for its magnetization.

${ }^{6}$ This scaling law can actually be true only for the singular part of $F$. The reason is that at the critical point one has $K_{L}=K_{c}$ and $h_{L}=0$ for all $L$ (see below) and therefore $F\left(K_{c}, 0\right)=L^{-3} F\left(K_{c}, 0\right)$, which is true only if $F\left(K_{c}, 0\right)$ is zero or $\infty$. In practice the singular part of $F$ is zero at the critical point (it is only the derivatives of $F$ which diverge at the critical point).

${ }^{7}$ I have chosen $\epsilon$ to be $K_{c}-K$ instead of $K-K_{c}$ so that $\epsilon$ is positive for temperatures above $T_{c}$.

${ }^{8}$ The original Ising interaction is invariant to the transformation $K \rightarrow K, h \rightarrow-h$, and $s_{\overrightarrow{\mathrm{n}}} \rightarrow-s_{\overrightarrow{\mathrm{n}}}$ for all $\overrightarrow{\mathrm{n}}$. This means that the free energy $F$ cannot depend on the sign of $h$, so it could be written as a function of $h^{2}$. One would expect this symmetry to be apparent in the effective Ising model; namely, one would expect that $K_{L} \rightarrow K_{L}, h_{L} \rightarrow-h_{L}$, and $s_{\mathrm{m}}^{\frac{1}{\mathrm{~m}}} \rightarrow-s_{\mathrm{m}}^{\frac{1}{\mathrm{~m}}}$ under this transformation. Hence $K_{L}$ should depend only on $h^{2}$ but $h_{L}$ should change sign when $h$ changes sign.

${ }^{9}$ The function $u\left(K_{L}, h_{L}^{2}\right)$ depends only on $h_{L}^{2}$ (i.e., is even in $\left.h_{L}\right)$ because $F\left(K_{L}, h_{L}\right)$ and $\xi\left(K_{L}, h_{L}\right)$ are even functions of $h_{L}$.

${ }^{10}$ In quantum field theory, where the renormalization group first arose, there is a corresponding analyticity problem; namely, the renormalized amplitudes of quantum electrodynamics diverge when the electron mass goes to zero yet it is assumed that the function (called $\psi$ in Gell-Mann and Low, Ref. 2) appearing in the renormalization-group equations has a finite limit for zero electron mass. The $\psi$ function was defined by Gell-Mann and Low in terms of renormalized amplitudes, so the assumption seems questionable. But Gell-Mann and Low showed that $\psi$ does have a zero-mass limit through fourth order in perturbation theory for which $\psi$ can be calculated explicitly. For further discussion see Gell-Mann and Low (Ref. 2) or Wilson (Ref. 2). This analyticity problem is ignored in Bogoliubov and Shirkov (Ref. 2).

${ }^{11} \mathrm{~L}$. P. Kadanoff (private communication). In the anisotropic Heisenberg model of a ferromagnet an irrelevant variable could be the ratio of the $\sigma_{x} \cdot \sigma_{x}$ nearestneighbor coupling to the $\sigma_{z} \cdot \sigma_{z}$ nearest-neighbor coupling. (See, however, Ref. 13.)

${ }^{12}$ In special cases equations like Eqs. (47) and (48) may have solutions behaving as $L^{y}$ and $L^{y} \ln L$ instead of $L^{y}$ and $L^{z}$. This cannot occur here (it is shown below that $z \neq y)$.

${ }^{13} \mathrm{An}$ example where this is relevant is in the theory of the Heisenberg ferromagnet, where the fixed point associated with the isotropic ferromagnet is expected to be unstable with respect to adding anisotropic interactions to the Hamiltonian as well as to changing the temperature or magnetic field. See D. Jasnow and M. Wortis, Phys. Rev. 176, 739 (1968); E. Riedel and F. Wegner, Z. Physik $\underline{225}, 195$ (1969), Phys. Rev. Letters $\underline{24}, 730$ (1970); $\underline{24}, 930$ (E) (1970).

${ }^{14}$ For an introduction to the theory of limit cycles, see N. Minorsky, Nonlinear Oscillations (Van Nostrand, Princeton, N. J., 1962), Chap. 3. See also K. Wilson, in Ref. 2. 